\title{
Statistics of superimposed flares in the Taurus molecular cloud ${ }^{\star}$
}

\author{
K. Arzner ${ }^{1}$, M. Güdel ${ }^{1}$, K. Briggs ${ }^{1}$, A. Telleschi ${ }^{1}$, and M. Audard ${ }^{2, \star \star ~}$ \\ 1 Paul Scherrer Institut, CH 5232 Villigen, Switzerland \\ e-mail: arzner@astro.phys.ethz.ch \\ 2 Columbia Astrophysics Laboratory, 550 West 120th St., MC 5247, New York, NY 10027, USA
}

Received 12 October 2006 / Accepted 19 February 2007

\begin{abstract}
Context. Stochastically occurring flares provide a possible mechanism of coronal heating in magnetically active stars such as T Tauri objects in star-forming regions.

Aims. We investigate the statistics of stellar X-ray light curves from the XMM-Newton Extended Survey of the Taurus Molecular Cloud (XEST).

Methods. To this end, the light curve is modeled as superimposed flares occurring at random times and with random amplitudes. The flare shape is estimated non-parametrically from the observations, while the flare amplitude distribution is modeled as a truncated power law, and the flare times are assumed as uniformly distributed. From these model assumptions, predictions on the binned counts are derived and compared with the observations.

Results. From a sample of 22 XEST observations matching the above model assumptions we find that the majority of cases have flare amplitude distributions with slopes steeper than two. This favours the role of small flares in coronal heating for 5 targets, of which, however, 4 are foreground or background main-sequence stars.
\end{abstract}

Key words. methods: statistical - stars: coronae - stars: pre-main sequence - X-rays: stars

\section{Introduction}

Stellar flares are violent manifestations of structural instabilities in stellar atmospheres, and often dwarf solar flares in terms of energy output and variability (Güdel 2004). Observationally, they are most pronounced in soft X-rays, where the luminosity may increase by many orders of magnitude during the flare. Stellar flares are believed to be related to magnetic fields, and are frequently observed in $\mathrm{T}$ Tauri stars and protostars (typical flare decay times are hours, and there may be several - observable - flares per day). Such objects have been the target of the XMM-Newton Extended Survey of the Taurus Molecular Cloud (XEST; Güdel et al. 2007) to which the present series of articles is devoted.

Some stars produce sporadic large flares which are easily recognized as such, but others are apparently in a state of continuous flaring activity, where most flares are too small to be resolved by photon counting observations. In the latter situation, numerous small flares are superimposed, resulting in fluctuations that cannot be explained by a Poisson process of constant intensity. The suggestion that a large population of randomly occurring small flares is producing much or all of the observed high-energy radiation from a corona was initially made for the solar corona based on observations (e.g., Lin et al. 1984; Hudson 1991) and theoretical concepts (Parker 1988). Observationally, the distribution of the radiative energy (or flare-peak power)

\footnotetext{
* Appendix A is only available in electronic form at http: //www . aanda. org

$\star \star$ Present address: Integral Data Centre, Ch. d'Ecogia 16, 1290 Versoix, Switzerland \& Geneva Observatory, University of Geneva, Ch. des Maillettes 51, 1290 Sauverny, Switzerland.
}

released in (hard or soft) X-rays has been found to obey a power law,

$$
\frac{\mathrm{d} N}{\mathrm{~d} E}=k E^{-\alpha}
$$

where $\mathrm{d} N$ is the number of flares per unit time with a total energy in the interval $[E, E+\mathrm{d} E]$, and $k$ is a constant. If $\alpha \geq 2$, then the energy integration (for a given time interval) diverges for the lower integration limit $E_{\text {min }} \rightarrow 0$, that is, by extrapolating the power law to sufficiently small flare energies, any energy release power can be attained. This is not the case for $\alpha<2$. Solar studies have repeatedly resulted in $\alpha$ values of 1.6-1.8 for ordinary solar flares (Crosby et al. 1993), but some more recent studies of low-level flaring suggest $\alpha=2.0-2.6$ (Krucker $\&$ Benz 1998; Parnell \& Jupp 2000).

The concept of stochastic flares heating coronae has found appeal also in the stellar case, in particular for magnetically active stars. The latter show properties that are difficult to explain with steady heating mechanisms but follow naturally from flare concepts: i) many magnetically active stars show coronal electron temperatures in excess of $10 \mathrm{MK}$, reminiscent of flaring plasma, with an emission measure distribution that can naturally be explained by the sum of emission measure distributions of randomly occurring flares (Güdel et al. 2003); ii) measured electron densities in active stars are elevated, often reaching values of several times $10^{10} \mathrm{~cm}^{-3}$ (Ness et al. 2004); iii) magnetically active stars are continuous sources of non-thermal radio emission, ascribed to gyrosynchrotron emission from accelerated electrons (Güdel 2002).

Interpretations of stellar X-ray emission in terms of stochastic flaring date back to the late eighties but have found renewed 
interest in particular with more recent satellite observations that allow for longer or more sensitive observations than hitherto possible. A summary of all previous observations has been given by Güdel (2004); we briefly summarize the results. A new methodology in flare identification was applied by Audard et al. (1999, 2000 ) to magnetically active, nearby main-sequence stars. They found a predominance of relatively steep power laws including $\alpha \geq 2$. Full forward modeling of a superposition of stochastic flares was applied to EUV and X-ray light curves by Kashyap et al. (2002) and Güdel et al. (2003) based on Monte Carlo simulations, and by Arzner \& Güdel (2004) based on an analytical formulation. These investigations converged to $\alpha \approx 2.0-2.5$ for $\mathrm{M}$ dwarfs. If the power-law flare energy distribution extends by about 1-2 orders of magnitude below the actual detection limit in the light curves, then the entire emission could be explained by stochastic flares.

Generally, larger flares are found to be harder than smaller flares (Güdel 2004), and the latter are harder than the quiescent emission; this leaves the possibility that the softest, quiescent emission could be due to a large number of small, unresolved superimposed flares. We shall, indeed, adopt here the working hypothesis that quiescent emission could be due to unresolved superimposed flares, and these would produce overall softer emission, as observed during "quiescence".

Young stellar objects such as T Tauri stars are extremely active X-ray sources, showing the same characteristics also found in active main-sequence stars. An extension of the stochasticflare studies to $\mathrm{T}$ Tauri stars is warranted, but the larger distances of these stars and consequently their lower fluxes have made such investigations much more difficult. Two studies have been undertaken, one by Wolk et al. (2005) on a sample of $\mathrm{T}$ Tauri stars in the Orion region observed by Chandra, and one by Stelzer et al. (2007) on a sample of T Tauri stars in the Taurus Molecular Cloud; the latter study includes a reconsideration of the sample presented by Wolk et al. The results of these investigations are not fully conclusive, with $\alpha$ values of $1.9 \pm 0.2$ and $2.4 \pm 0.5$ for the Orion and the Taurus sample, respectively (Stelzer et al. 2007).

The present work attempts to extend theoretical and numerical work presented by Arzner \& Güdel (2004) for mainsequence stars to a sample of T Tauri stars in the Taurus region.

\section{Methods}

Scargle (1998) has proposed a method to find changes in the count rates and thus decompose the observed light curve into Bayesian blocks of piecewise constant count rate. The Bayesian block method is applied to XEST data in an accompanying article (Stelzer et al. 2007).

While the Bayesian blocks are successful in detecting abrupt changes in the count rate, the assumption of piecewise constant flux is somewhat artificial and not well adapted to stellar flares, which typically have a rapid rise followed by a slow decay. In order to investigate the occurrence of flares down to very low levels, we now study an alternative model in which a constant flare shape $\xi(t)$ is assumed, and suppose that a flaring light curve is a superposition of many similar events. Mathematically, the flare times $t_{k}$ are assumed to be uniformly distributed with rate $\lambda$ [flares/s], and the light curve is modeled by a stationary random process of the form

$$
f(t)=\sum_{k} a_{k} \xi\left(t-t_{k}\right) \quad[\mathrm{ct} / \mathrm{s}]
$$

where the $a_{k}>0$ are flare amplitudes [cts/flare] drawn from some probability density $P\left(a_{k}\right)$. By definition, the flare amplitudes $a_{k}$ have units of counts (per flare), and the flare shape $\xi(t)$ has units $\mathrm{s}^{-1}$. All $a_{k}$ and $t_{k}$ are assumed to be statistically independent, and the observed photon arrival times are assumed to form a non-homogeneous Poisson process with intensity $f(t)$. The flare profile is normalized to one $\left(\int \xi(t) \mathrm{d} t=1\right)$ and we also require that

$\int t \xi(t) \mathrm{d} t<\infty$

The condition (Eq. (3)) is a technicality which will facilitate the estimation of the flare shape. It is valid in the frequently observed case of approximately exponentially decaying flares.

The assumptions of independence and linear superposition expressed by Eq. (2) not only lead to a simple expression ${ }^{1}$ for the power spectral density (or two-time function)

$$
\begin{aligned}
|\hat{f}(\omega)|^{2} & =|\hat{\xi}(\omega)|^{2}\left|\sum_{k=1}^{N} a_{k} \mathrm{e}^{\mathrm{i} \omega t_{k}}\right|^{2} \\
& \stackrel{N \rightarrow \infty}{\longrightarrow}|\hat{\xi}(\omega)|^{2}\left\{\lambda^{2}\langle a\rangle^{2} \delta(\omega)+\lambda\left\langle a^{2}\right\rangle\right\}
\end{aligned}
$$

but also admit a closed-form representation of the single-time distribution of $f(t)$ in terms of characteristic functions (i.e., the Fourier transforms of probability densities, see Lukacs 1970). In particular, Arzner \& Güdel (2004) have shown that given the characteristic function $\phi_{a}(s)$ of the flare amplitudes $a_{k}$ and the characteristic function $\phi_{F}(s, \Delta t)$ of the bin content (Poisson parameter) $F(t)=\int_{t}^{t+\Delta t} f\left(t^{\prime}\right) \mathrm{d} t^{\prime}$ are related by

$\phi_{F}(s, \Delta t)=\exp \left(-\lambda \int_{-\infty}^{\infty} \mathrm{d} t\left(1-\phi_{a}[s \Xi(t, \Delta t)]\right)\right)$,

where $\Xi(t, \Delta t)=\int_{t}^{t+\Delta t} \xi\left(t^{\prime}\right) \mathrm{d} t^{\prime}$ is the flare shape convolved with the observational time bin. The derivation of Eq. (5) is discussed in detail in Arzner \& Güdel (2004) and exploits the independence of flare times and -amplitudes, which allows the factorization of the characteristic function, and a decomposition into the possible (Poisson distributed) numbers of flares occurring during the observation. Notice that in Eq. (4) it is tacitly assumed that both $\langle a\rangle$ and $\left\langle a^{2}\right\rangle$ exist.

Several observational predictions can be derived from Eq. (5), such as the distribution $P_{\Delta}(n)$ of counts in bins of given duration $\Delta t$ (i.e., the distribution of the values of the light curve). The detailed calculations are given in Arzner \& Güdel (2004), and result in

$P_{\Delta}(n)=(2 \pi)^{-1} \int_{0}^{2 \pi} \mathrm{d} s \mathrm{e}^{-\mathrm{i} n s} \phi_{F}\left(i-i \mathrm{e}^{\mathrm{i} s}, \Delta t\right)$.

An additive constant background $b$ [ct s$~^{-1}$ ] is easily included, as it amounts to replacing $\phi_{F}(s, \Delta t)$ by $\mathrm{e}^{\mathrm{i} s b \Delta t} \phi_{F}(s, \Delta t)$. In principle, part of the "quiescent" emission could be truly constant, i.e., not due to superimposed flares. Our analysis method cannot determine such a contribution separately. However, as outlined in the introduction, our assumption is that there is no separate quiescent contribution to the light curve, but that "quiescent" emission is due to small, unresolved, superimposed flares. In

${ }^{1}$ This follows from $f(t)$ being the convolution product of $\xi(t)$ and $\sum_{k} a_{k} \delta\left(t-t_{k}\right)$. The limit in Eq. (4) applies to sums over infinitely many flares. See Bondesson (1988) for a general introduction and Mitra-Kraev \& Benz (2001) for a solar application. 
Arzner \& Güdel (2004), Eq. (6) (and a similar result for the photon waiting time distribution) have been applied to EUVE data of $\mathrm{AD}$ Leo. In this article, we apply Eq. (6) to XMM-Newton data, and introduce an important methodical refinement. In the original work, the flare shape was taken ad-hoc as a one-sided exponential, with a decay constant chosen by eye. Here we estimate the flare shape empirically using Eq. (4).

It should be pointed out that the application of Eq. (6) represents a rather drastic form of data reduction, since it projects away the time ordering of the observed light curve. The presence of the flares manifests only in the deviation of the binned count histograms from pure Poisson distributions. The advantage is that the method is insensitive to data gaps and does not require the flares to be resolved within the counting statistics. It is, in fact, especially adapted to faint but flaring sources.

\section{Observations and data reduction}

\subsection{Data}

The data used for our investigation are part of XEST (Güdel et al. 2007), a project that investigates X-ray emission of a large sample of $\mathrm{T}$ Tauri stars and protostars in the Taurus star-forming region using the XMM-Newton X-ray observatory (Jansen et al. 2001). The survey comprises 27 different fields across the cloud, and most of them used exposure time of approximately $30 \mathrm{ks}$ although a few were exposed up to $\approx 130 \mathrm{ks}$. The survey makes use of the European Photon Imaging Cameras (EPIC) of the MOS (Turner et al. 2001) and the PN type (Strüder et al. 2001).

The XMM-Newton observatory uses grazing incidence mirrors for X-ray imaging in the range of 0.2 to $10 \mathrm{keV}$. The Xray photons are detected by two MOS-type CCD arrays and a pn-type CCD array. The time resolution of these arrays depends on their type and also on the operation mode. For the observations considered here, the MOS-type arrays (Turner et al. 2001) have a time resolution of $2.6 \mathrm{~s}$ whereas the pn array has a time resolution $0.07 \mathrm{~s}$. In order to improve the statistics we have coadded all available MOS and pn data. In order to avoid artificial fluctuations, all detectors are required to be simultaneously operational. Since we are interested in light curves, we accept all energies between 0.5 and $7.3 \mathrm{keV}$. This choice of energies is motivated by instrumental considerations and by the uniformity of data treatment.

From all XEST observations we have selected a set where the model assumption of Eq. (2) is plausible by inspection of the light curves and where $>1000$ counts are available (but pile-up can be neglected), and have determined the maximum-likelihood parameters of the flare amplitude distribution. In the rest of this section, we discuss the detailed procedure considering as an example the observation HD 31305 (XEST-26-051), which is a A0 V background star, the X-ray emission of which probably comes from an unseen companion. The raw data of this observation are presented in Fig. 1. The top panel shows the photon arrival time versus energy (one dot represents one count). The bottom panel shows the energy-integrated, time-binned counts. The time bins $\Delta t$ are $2^{-n}$-th fractions of the total observing time, as needed for the Fourier analysis of the pulse shape (Sect. 3.2), and are chosen such that the bins contain of order 10 counts. Different observations use different time bins. A background estimate has been obtained from $\sim 10$ times larger source-free extraction regions, giving a (scaled, constant) background rate of $b=0.0027 \mathrm{ct} \mathrm{s}^{-1}$ (this value refers to HD 31305). Since the relative error of $b$ is only about $0.2 \%$, we consider $b$ as exactly known.
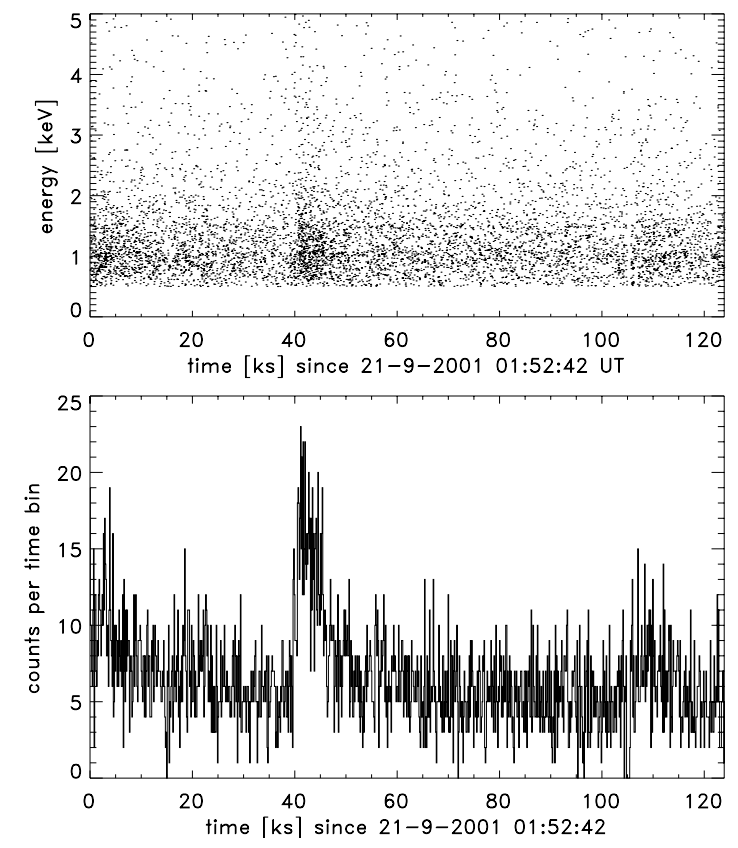

Fig. 1. Top: photon arrival time versus energy of HD 31305 the (XEST observation 26-051). Bottom: energy-integrated light curve. The time bin size is $124 \mathrm{~s}$.

\subsection{Estimation of the flare profile}

In order to estimate the flare profile $\xi(t)$ from the observations, we work with the power spectrum (or, equivalently, the autocorrelation), thus making use of the assumption of stationarity. Consider Eq. (4). The factor in curly brackets, representing the random flare pulses, is constant except at $\omega=0$. On the other hand, the factor $|\hat{\xi}(\omega)|$, representing the flare shape, is continuous at $\omega=0$ and satisfies $|\hat{\xi}(0)|=1$ (both by virtue of Eq. (3)). Therefore, $|\hat{\xi}(\omega)|$ can be obtained from $|\hat{f}(\omega)|$ by continuous interpolation to $\omega=0$ and appropriate scaling. This is the basic idea used here to estimate the flare profile; however, the actual implementation requires two additional steps.

First, $|\hat{f}(\omega)|$ must be estimated from the observed counts. To this end we consider the power spectrum $\left|\hat{n}_{i}\right|$ of the binned light curve, assuming that the bins are sufficiently fine to resolve the flare shape. (In practice, the power spectrum $\left|\hat{n}_{i}\right|$ is computed by a fast Fourier transform and we use a discrete frequency index to indicate the actual numerical implementation. The Fourier normalization convention is that $\hat{n}_{0}$ equals the total number of observed counts.) $\left|\hat{n}_{i}\right|$ represents a noisy version of $\left|\hat{f}_{i}\right|$, involving two kinds of noise. The first type is photon counting noise. Since the counts $n_{i}$ in bins of content $F_{i}$ satisfy $\left\langle n_{i} n_{j}\right\rangle=F_{i} F_{j}+F_{i} \delta_{i j}$ (see, e.g., Feller 1968 and Reiss 1993), the power spectral densities of the binned events and of the bin contents are related by $\left|\hat{n}_{i}\right|^{2}=\left|\hat{F}_{i}\right|^{2}+\mathcal{N}$, where $\mathcal{N}$ is the expected total number of counts. The photon counting noise thus manifests in a constant additive contribution to the power spectrum. The second type of noise stems from the finite number of observed flares, and will be referred to as flare shot noise. As a consequence, the term in curly brackets in Eq. (4) becomes a fluctuating function of frequency. For the discrete Fourier representation, the fluctuations are approximately exponentially distributed with variance $N\left\langle a^{2}\right\rangle$ (this was found numerically). Thus, the flare shot noise is a multiplicative noise in frequency space with a relative amplitude of unity. Both the flare-shot and photon counting noise can be suppressed by filtering. The flare-shot noise, which is 
multiplicative in the frequency domain, is removed by filtering $\ln \left|\hat{n}_{i}\right|^{2}$. The photon counting noise, which is white in the time domain, is removed by filtering $\xi(t)$ once this is obtained from a Fourier back transform (see below). Our filters are implemented as Lee filters (Lee 1986) with sizes adapted to the expected noise, and the results are tested by eye for compatibility with the observed light curve.

Secondly, and more fundamentally, the spectrum $|\hat{\xi}(\omega)|$ does not contain the phase information needed to Fourier-invert $|\hat{\xi}(\omega)| \mathrm{e}^{\mathrm{i} \phi(\omega)}$ into the flare shape $\xi(t)$. We shall not address here the general phase retrieval problem (Klibanov et al. 1995) but make the minimal phase assumption (Burge et al. 1974)

$\phi(\omega)=\frac{1}{\pi} \int_{-\infty}^{\infty} \frac{\ln |\hat{\xi}(s)|}{\omega-s} \mathrm{~d} s$,

which is equivalent to requiring $\xi(t)=0$ for $t<0$. Such causal flare shapes apply to flares with a rapid (unresolved) rise phase, followed by a slower decay. The integral in Eq. (7) is understood in the principal value sense and can be computed by a discrete Hilbert transform (Henery 1984).

The full procedure of estimating $\xi(t)$ from the observations is illustrated in Fig. 2. The gray crosses in the top panel represent the modulus of the fast Fourier transform of the light curve of Fig. 1 (bottom), using 1024 equal time bins of duration $124 \mathrm{~s}$. The zero frequency signal clearly peaks out, as expected from Eq. (4). The Poisson noise level is indicated by white dashed line. The solid curve in Fig. 2 (top) represents our filtered estimate for $|\hat{f}(\omega)|$. This is then scaled to $|\hat{\xi}(\omega)|$, endowed with the minimal phase of Eq. (7), and transformed back into time domain to obtain the estimate for the flare shape (Fig. 2 bottom, black line). For comparison, the result of using $\phi(\omega)=0$ is also shown (gray line); it represents the convolution square root of the autocorrelation.

When treating the full set of observations (Table 1 below), the flare shape is estimated individually for each observation. Different observations have thus flare shapes of different decay time. Within a given observation, the flare shape is assumed to be constant.

\subsection{Determination of the flare rate and amplitude distribution}

Once the flare profile is known, we numerically evaluate Equation (6) for a power-law flare amplitude distribution of the form

$P\left(a_{k}\right)=\left\{\begin{array}{cl}c a_{k}^{-\alpha} & A \leq a_{k} \leq B \\ 0 & \text { else }\end{array}\right.$

where $A$ and $B$ (in units of counts per flare) are lower and upper cutoffs, respectively, and $c$ is a normalization constant. The cutoffs $A$ and $B$ must in general be applied in order to ensure that $P\left(a_{k}\right)$ can be normalized and that the first moments exist, as assumed in Eq. (4). For probabilistic normalization, a lower cutoff is needed if $\alpha \geq 1$ and an upper cutoff is needed if $\alpha<1$. Furthermore, the existence of moments up to second order requires an upper cutoff if $\alpha<3$. In order to be free of theoretical restrictions on $\alpha$ we assume that both lower and upper cutoffs exist, which we parameterize technically by $A$ and $B / A$. We then determine the parameters $(\alpha, A, B)$ so that they maximize the Poisson likelihood of the observed distribution of the binned counts. The flare rate $\lambda$ is determined by the normalization constraint $\hat{\xi}(0)=1$, implying that $\lambda\langle a\rangle+b=\hat{f}(0)$. The power law index and dynamical range of the flare amplitudes are limited to $0.5 \leq \alpha \leq 5$ and $10^{2} \leq B / A \leq 10^{6}$; this choice
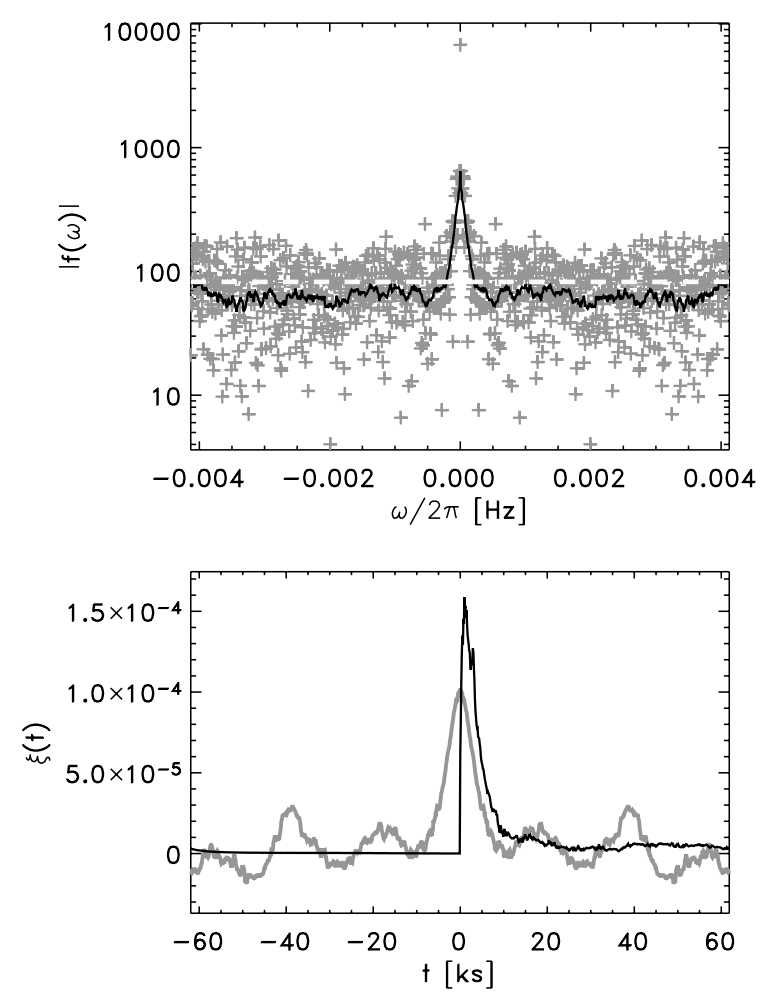

Fig. 2. Estimation of the flare shape of Fig. 1. Top: observed (crosses) and filtered (solid line) spectral densities. The Poisson noise level $\mathcal{N}$ is indicated by white dashed line. Bottom: flare shape obtained from the minimum-phase (black) and zero-phase (gray) assumptions. It is the minimum-phase solution which is used in all further analysis.

covers the physically expected and observationally distinguishable situations. The lower cutoff $A$ is not constrained since arbitrarily small flares are possible. The best-fit solution for $P_{\Delta}(n)$ for the data of Figs. 1 and 2 (HD 31305) is shown in Fig. 3, where the black histogram represents the data, and the red histogram represents the best-fit model. The insets represent projections of the likelihood surfaces at $(0.68,0.90,0.99)$ confidence levels, obtained by thresholding the (Poisson) likelihood ratio of the predicted histograms relative the the maximum-likelihood solution. The best-fit solution is marked by crosses, and equals $A=0.0058$ counts/flare and $\alpha=2.05$. The (projected) $68 \%$ confidence errors of the power law index is $1.99 \leq \alpha \leq 2.23$. It should be noticed that the choice of $68 \%$ confidence is ad hoc, and favours small error bars. If we had used $95 \%$ confidence levels (between the light gray and medium gray regions in the inlets of Fig. 3), the corresponding error of the power law index was found to be $1.93 \leq \alpha \leq 2.32$. Note from Fig. 3 that the limits of $\alpha$ and $A$ are not constrained by the data but limited by the explored range of $A$. The most likely flare rate, derived from the most likely $(\alpha, A, B)$, is $\lambda=0.98$ flares/s. We recall that it is the deviation of the observed curve in Fig. 3 from a purely Poisson shape which reflects the presence of the flares, and is detected by our method. The case of quiescent emission, where the Poisson intensity $f(t)$ is constant, can be obtained as a special limit of Eq. (4) when $\lambda \rightarrow \infty$ (Sect. 3.5).

\subsection{Monte-Carlo exploration}

In order to speed up the parameter space exploration and to extend the explored parameter range, we use a combination of 
Table 1. Maximum-likelihood parameters with (projected) $68 \%$ confidence intervals. Asterisks denotes error bars which are so large that they could not be determined. $N_{\mathrm{cnt}}$ is the number of observed counts; $\mathcal{N}_{\mathrm{bg}}$ is their expected background contribution. The parameters $(\alpha, A, B)$ are defined in Eq. (8); $A$ is in units of counts/flare. $\lambda$ is the flare rate [flares/s] and is derived from $(\alpha, A, B)$ and the observed average count rate. The values of this table refer to Figs. 4 and A.1 to A.3. The stellar type is: type $1=$ protostar, type $2=$ accreting T Tau star (classical T Tauri star), type 3 = non-accreting T Tau star (weak line T Tauri star; we note that the classification can be ambiguous in some cases, see Güdel et al. 2007); "MS" refers to main sequence stars that are not recognized members of the Taurus Molecular Cloud. The last column shows the best-fit reduced chi square; observations with $\chi_{\text {red }}^{2}>2$ represent bad fits; observations with $\chi_{\text {red }}^{2}<1$ hint at noisy data where the statistics is not sufficient to discriminate between different model parameters.

\begin{tabular}{|c|c|c|c|c|c|c|c|c|c|}
\hline Source name & type & XEST\# & $N_{\mathrm{cnt}}$ & $\mathcal{N}_{\mathrm{bg}}$ & $A$ [cts/flare] & $\alpha$ & $B / A$ & $\lambda$ [flares/s] & $\chi_{\mathrm{red}}^{2}$ \\
\hline test & - & UNIFOR & 9996 & 1000.0 & $5.7 \times 10^{-1_{*}^{*}}$ & $3.3_{*}^{*}$ & $3.6 \times 10^{2 *}$ & $8.8 \times 10^{-2}{ }_{1.2 \times 10^{-2}}$ & 0.58 \\
\hline V807 Tau & 2 & 04-012 & 2672 & 38.1 & $1.1_{*}^{4.1 \times 10^{1}}$ & $0.7_{*}^{*}$ & $5.8 \times 10^{1 *} *$ & $3.7 \times 10^{-3}{ }_{1.5 \times 10^{-3}}^{1.1}$ & 1.15 \\
\hline GK Tau & 2 & 04-035 & 2471 & 246.7 & $9.1 \times 10^{-2}{ }_{*}^{8.2}$ & $3.0_{*}^{*}$ & $1.2 \times 10^{5 *}$ & $4.1 \times 10^{-1}{ }_{6.9 \times 10^{-3}}^{*}$ & 0.51 \\
\hline L1489 IRS & 1 & 06-059 & 3027 & 303.4 & $1.2 \times 10_{*}^{12.1 \times 10^{1}}$ & $4.7_{*}^{*}$ & $1.5 \times 10^{4}{ }_{*}^{*}$ & $6.2 \times 10^{-3} \frac{1.6 \times 10^{1}}{3.4 \times 10^{-3}}$ & 1.16 \\
\hline 2MASS J04345693+2258 & MS & $08-003$ & 3215 & 168.9 &  & $0.5_{*}^{0.7}$ & $5.2 \times 10^{5_{*}^{*}}$ & $6.1 \times 10^{-4}{ }_{4.8 \times 10^{-4}}^{9.9 \times 10^{-4}}$ & 5.11 \\
\hline 2MASS J04351316+2259 & MS & 08-014 & 1199 & 163.0 & $1.2 \times 10_{4.8}^{12.0 \times 10^{1}}$ & $4.4_{2.9}^{*}$ & $4.0 \times 10^{2 *} *$ & $1.5 \times 10^{-3} \frac{2.8 \times 10^{-3}}{9.7 \times 10^{-4}}$ & 2.08 \\
\hline HD 29050 & MS & 08-017 & 1299 & 66.3 & $1.8 \times 10^{1} \underset{1.1 \times 10^{1}}{2.5 \times 10^{1}}$ & $5.0_{3.8}^{*}$ & $4.1 \times 10_{2.1 \times 10^{3}}^{3}$ & $1.3 \times 10^{-3}{ }_{9.2 \times 10^{-4}}^{2.0 \times 10^{-3}}$ & 1.78 \\
\hline HQ Tau & 3 & 08-037 & 6616 & 275.7 & $1.6 \times 10_{8.0}^{1} \underset{2.2 \times 10^{1}}{2}$ & $5.0_{3.5}^{*}$ & $1.9 \times 10^{2 *}$ & $9.8 \times 10^{-3} \begin{array}{l}1.6 \times 10^{-2} \\
7.1 \times 10^{-3}\end{array}$ & 3.69 \\
\hline HP Tau & 2 & 08-048 & 4263 & 1163.9 & $1.7 \times 10_{*}^{-1}{ }_{*}^{1.7 \times 10^{1}}$ & $2.4_{*}^{*}$ & $7.1 \times 10^{5 *}$ & $2.0 \times 10^{-1} \begin{array}{c}1.8 \times 10^{1} \\
5.0 \times 10^{-3}\end{array}$ & 1.48 \\
\hline HP Tau G2 & 3 & $08-051$ & 19521 & 34.6 & $1.2 \times 10_{*}^{-17.4}$ & $3.0_{*}^{*}$ & $4.0 \times 10^{1}{ }_{*}^{*}$ & $3.1_{7.4 \times 10^{-2}}^{7.7 \times 10^{2}}$ & 1.67 \\
\hline CoKu LkHa 332 G2 & 3 & $10-017$ & 5373 & 87.0 & $2.5_{*}^{1.1 \times 10^{1}}$ & $3.3_{*}^{*}$ & $1.3 \times 10^{1 *}{ }_{*}^{*}$ & $4.6 \times 10^{-2}{ }_{1.3 \times 10^{-2}}$ & 1.54 \\
\hline DN Tau & 2 & $12-040$ & 7464 & 149.1 & $3.0 \times 10^{-1}{ }_{*}^{4.6}$ & $3.0_{*}^{*}$ & $9.0 \times 10^{2 *}{ }_{*}^{*}$ & $4.3 \times 10^{-1}{ }_{4.1 \times 10^{-2}}^{*}$ & 0.45 \\
\hline $\mathrm{CoKu} \mathrm{Tau} / 3$ & 3 & $12-059$ & 18640 & 619.3 & $1.7 \times 10_{*}^{-1}{ }_{*}^{3.6}$ & $2.9_{*}^{*}$ & $1.6 \times 10^{1 *}{ }_{*}^{*}$ & $1.9_{1.3 \times 10^{-1}}^{*}$ & 1.15 \\
\hline DI Tau & 3 & $15-042$ & 3305 & 1281.0 & $2.6_{*}^{2.2 \times 10^{1}}$ & $3.0_{*}^{*}$ & $2.1 \times 10^{1 *}{ }_{*}^{*}$ & $2.1 \times 10^{-2}{ }_{3.5 \times 10^{-3}}$ & 0.60 \\
\hline IT Tau & 2 & $18-030$ & 15736 & 312.0 & $3.6 \times 10_{*}^{-1}{ }_{*}^{1.0 \times 10^{1}}$ & $2.9_{*}^{*}$ & $2.0 \times 10^{1 *}$ & $8.3 \times 10_{4.2 \times 10^{-2}}^{-1}$ & 1.01 \\
\hline Anon 1 & 3 & $20-005$ & 8041 & 473.3 & $2.1 \times 10^{-1}{ }_{*}^{2.3 \times 10^{1}}$ & $1.8_{*}^{*}$ & $5.0 \times 10^{2 *}$ & $1.3 \times 10^{-1}{ }_{7.9 \times 10^{-3}}^{5.8}$ & 1.72 \\
\hline V773 Tau & 3 & $20-042$ & 37324 & 134.4 & $1.1 \times 10^{-1}{ }_{*}^{1.9}$ & $3.5_{*}^{*}$ & $9.0 \times 10^{1 *}$ & $6.6_{4.6 \times 10^{-1}}^{*}$ & 0.76 \\
\hline 1AXG J041453+2805 & MS & $20-071$ & 3026 & 242.2 & $3.7 \times 10_{*}^{-2.4}$ & $3.1_{*}^{*}$ & $1.8 \times 10^{5 *}$ & $1.2_{2.8 \times 10^{-2}}^{*}$ & 0.79 \\
\hline JH 188 & MS & $22-006$ & 1818 & 205.0 & $7.4_{*}^{1.9 \times 10^{1}}$ & $3.6_{*}^{*}$ & $3.7 \times 10^{3 *}{ }_{*}^{*}$ & $2.4 \times 10^{-3}{ }^{3.3 \times 10^{-1}} 1.1 \times 10^{-3}$ & 2.33 \\
\hline HD 285845 & MS & $22-024$ & 52074 & 864.7 & $3.8 \times 10^{1}{ }_{2.6 \times 10^{1}}^{4.5 \times 10^{1}}$ & $4.6_{3.7}^{*}$ & $9.4 \times 10^{4}{ }_{*}^{*}$ & $1.9 \times 10^{-2} \underset{1.6 \times 10^{-2}}{2.4 \times 10^{-2}}$ & 3.94 \\
\hline HL Tau & 1 & $22-043$ & 2956 & 252.4 & $9.6 \times 10^{-2} *$ & $3.0_{*}^{*}$ & $2.5 \times 10^{2 *}$ & $2.8 \times 10^{-1}{ }_{1.0 \times 10^{-2}}$ & 0.81 \\
\hline V710 Tau & 2 & $22-070$ & 4701 & 442.7 & $4.0 \times 10_{*}^{-2} * 1.0 \times 10^{1}$ & $0.6_{*}^{*}$ & $2.4 \times 10^{2 *}$ & $2.4 \times 10^{-2}{ }_{5.5 \times 10^{-3}}$ & 3.85 \\
\hline HD 31305 & MS & $26-051$ & 7049 & 436.3 & $1.0 \times 10^{-3}{ }_{4.4 \times 10^{-4}}^{2.3}$ & $2.0_{1.9}^{2.5}$ & $6.4 \times 10_{4.4 \times 10^{2}}^{5^{*}}$ & $3.7_{7.6 \times 10^{-3}}^{6.3}$ & 0.85 \\
\hline
\end{tabular}

Monte-Carlo exploration and interactive search for an initial guess using a graphical user interface. Four representative results are illustrated in Fig. 4. The first line shows the same observation as Figs. 1 to 3, and is repeated here in order to clarify the presentation of Monte-Carlo results. The left column shows the light curve in the same binning as used in Eq. (6). Red dashed lines indicate the range of binned counts used for parameter estimation. Low counts are rejected when they interfere with data gaps due to increased background. The middle column shows the observed (black) and best-fit (red) histograms of binned counts. The $y$-axis represents the square root of the counts rather than the counts themselves; by this trick, the Poisson error becomes approximately 0.5 for all bins, so that the agreement between observation and model can be judged by visual inspection. The right column shows the Monte Carlo samples in $(A, \alpha)$-space (projection). Black dots denote samples that are accepted within $68 \%$ confidence; gray dots represents samples that are rejected. The best-fit solution is marked by large crosses. Different data sets use different time bins $\Delta t$. Note that since we show projected acceptance regions, black and gray dots may co-exist at a given location.

\subsection{Benchmark with non-flaring data}

As a benchmark, we have applied the method of Sect. 2 to a non-flaring data simulated from constant $f(t)$. The outcome is shown in the last line of Fig. 4. The distribution of binned counts follows a Poisson distribution (middle panel), whereas the flare shape, estimated by the procedure of Sect. 3.2 is approximately $\delta$-peaked (not shown). As can be seen from the right panel, the spectrum parameters $(A, \alpha)$ are not well constrained by the (simulated) observation. The same holds for the dynamic range $B / A$. The shape of the acceptance region in $(A, \alpha, B / A)$-space (Fig. 4 bottom right shows the $68 \%$ region) is determined by the flare amplitude distribution (Eq. (8)) and the procedure of Sect. 3.2. Since the mean count rate is adjusted by the choice of $\lambda$, the acceptance of models is determined by the higher order statistics, in particular by the variance of the expected bin contents, which becomes noticeable when it exceeds the Poisson (counting) noise of individual bins. As a result, models predicting few large flares (large $A$, small $\alpha$ ) are excluded and models predicting many small flares (small $A$, large $\alpha$ ) are accepted. In fact, a constant Poisson intensity $f(t)$ may be obtained from Eqs. (2) 


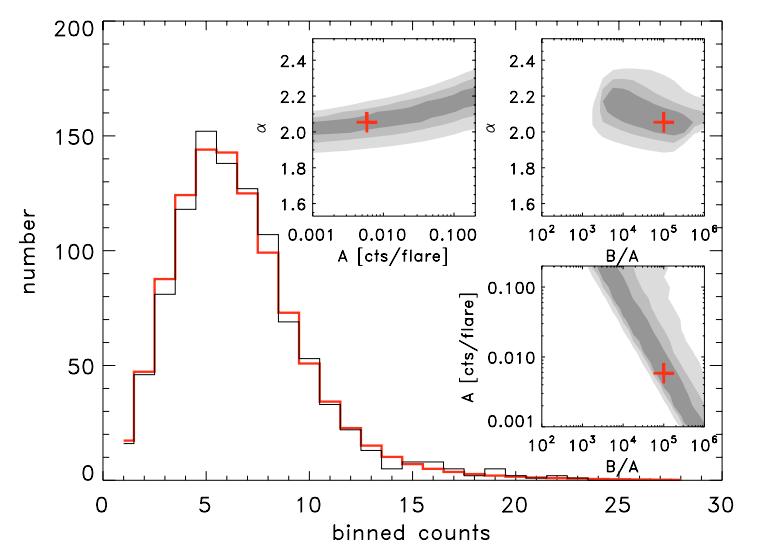

Fig. 3. Determination of best-fit parameters $(\alpha, A, B)$ of the flare amplitude distribution $P\left(a_{k}\right) \sim a_{k}^{-\alpha}$ with $A<a_{k}<B$ for HD 31305. The flare amplitudes $a_{k}$ and their lower and upper cutoffs $A$ and $B$ are in observed counts per flare.

and (8) as the limit of infinitely many $(\lambda \rightarrow \infty)$ infinitely small $\left(\left\langle a_{k}\right\rangle \rightarrow 0\right)$ flares, such that $\lambda\left\langle a_{k}\right\rangle$ equals the observed average (source) count rate. The boundary of the acceptance region in Fig. 4 (bottom right panel) is roughly given by the condition that the variance of the expected bin content, $\left\langle F^{2}\right\rangle-\langle F\rangle^{2}$, equals the Poisson variance $\langle F\rangle$. To summarize, quiescent light curves $(f(t)=$ const) allow only one parameter to be determined (the average count rate $\lambda\langle a\rangle)$ and do not constrain the individual parameters $(A, \alpha, A / B)$ as long as the predicted fluctuations of $f(t)$ are within the Poisson noise of the observation; the resulting acceptance region in $(A, \alpha)$-projection is open toward large $\alpha$ and small $A$.

\section{Results and discussion}

We have applied the procedure of Section 3 to a set of 22 XEST observations chosen to meet the model assumptions of a statistically homogeneous superposition of random-amplitude flares. The results are listed in Table 1 and illustrated in Fig. 4 and in the Appendix. All Monte Carlo explorations shown in these Figures involve about 5000 samples. The first line of Fig. 4 has already been discussed in Sect. 3.

The second and third lines of Fig. 4 show examples (2MASS J04351316+2259; XEST-08-014) of a strongly variable but photon-starved light curve, as well as an example where no visible flares are present (CoKu Tau/3; XEST-12-059). In both cases, the observed histogram (middle column, black) can be well reasonably represented by the model (middle column, red). In the case of 2MASS J04351316+2259 we may conclude that $\alpha>3$. In the case of $\mathrm{CoKu} \mathrm{Tau} / 3$, no such conclusion is possible, and the data are compatible with pure Poisson noise (as apparent from the light curve). Among the remaining Monte-Carlo results (Appendix, Figs. A.1 to A.3) we emphasize the following ones:

- GK Tau (XEST-04-035): Although the flare amplitude distribution cannot be tightly constrained by the data, good fits (middle column) are possible.

- XEST-08-003: this figure is shown as an example that cannot be fitted with the present model (the reduced chi square being 5.11), and is shown for comparison only. In fact, the light curve is not statistically homogeneous but shows enhanced activity for times $t<15 \mathrm{ks}$. Equally bad fits were found in two further investigated observations (XEST22-097 and XEST-22-100) which are thus not included in Table 1. Among the observations retained in Table 1, HQ Tau, HD 285845, and V710 Tau have large reduced chi squares as well, which reflect in systematic deviations between observed and predicted binned count histograms.

- HP Tau G2 (XEST-08-051): this is a relatively quiet observation with many data gaps and low background. Although the power law index is not constrained by the data, we may visually distinguish two "branches" of solutions: $A>1 \mathrm{cts} /$ flare and $\alpha>3$ or $A<1 \mathrm{cts} /$ flare and $\alpha<3$. Thus, based on the data we can make statements of the form: if the flares yield at least one count $(A>1 \mathrm{cts} /$ flare $)$ then $\alpha>3$.

- HD 285845 (XEST-22-024): Here, the power law index $\alpha$ and the lower cutoff $A$ can be constrained by the data according to $\alpha>3.7$ and $A \geq 26$ cts/flare.

- JH 188 (XEST-22-006): similar to HP Tau G2, in that $\alpha$ values above 3 require lower flare amplitude cutoffs $A$ below a few counts per flare. A more detailed analysis shows that an $\alpha$ value below 3 requires a dynamic range $B / A<10^{3}$.

As a general trend, one can see from Figs. 4 to A.3 that $A$ is correlated with $\alpha$. This correlation reflects the fact that the total number of counts (the integral under the power law distribution) should agree with the observed number of counts within Poisson errors. Thus, steep power laws ( $\alpha$ large) correspond to large lower cutoffs ( $A$ large).

The results of the other observations are summarized in Table 1. The targets we are studying in the present work are predominantly $\mathrm{T}$ Tauri stars identified in the Taurus Molecular Cloud (TMC), although two objects are classified as protostars, and we also include a few favorable X-ray targets found in the XEST survey that appear to be foreground or background stars. The TMC members have been classified according to the equivalent width of the $\mathrm{H} \alpha$ line (an accretion signature) and based on the presence of an infrared excess (a disk signature). We used the classification as tabulated in Güdel et al. (2007), i.e., type 1 objects are protostars, type 2 objects are accreting T Tauri stars ("classical T Tauri stars"), and type 3 objects are non-accreting T Tauri stars ("weak-line T Tauri stars"). The classification into accretors and non-accretors is, for this article, motivated by potential differences in their magnetic configurations. In Table 1, $N_{\text {cnt }}$ is the total number of counts present in the observation; this includes an estimated background contribution $\mathcal{N}_{\text {bg }}$. Note that $A$ values below one count per flare imply that the smallest postulated flares cannot be individually observed. The dynamic ranges $B / A$ cover usually between 2 and 4 orders of magnitude. The (derived) flare rates $\lambda$ are mostly in the order of one flare per kilosecond. In order to decide on whether confidence limits can be given on parameters, we use the criterion that there should be at least $\sqrt{N_{\mathrm{MC}}}$ rejected solutions (dots) outside the accepted parameter interval in a Monte-Carlo simulation of $N_{\mathrm{MC}}$ samples. Otherwise, we conclude that the confidence boundary is outside the explored parameter range, or that the parameter cannot be bound at all. In cases where the above criterion is not fulfilled, confidence limits are not given in Table 1 but the presence of large error bars is marked by asterisks. Inspection of Figs. A.1 to A.3 and of Table 1 shows that from all observations, $\alpha$ and $A$ can only be constrained in a few cases. For HD 31305, both upper and lower limits can be given, and $\alpha$ is tightly constrained between 1.9 and 2.5. The reduced chi square (1.03) indicates that the fit is acceptable. For XEST-08-014, only a lower limit on $\alpha$ can be given, $\alpha>2.9$. A similar finding holds for HD 29050, where $\alpha>3.8$, and HQ Tau, where $\alpha>3.5$, although in these 

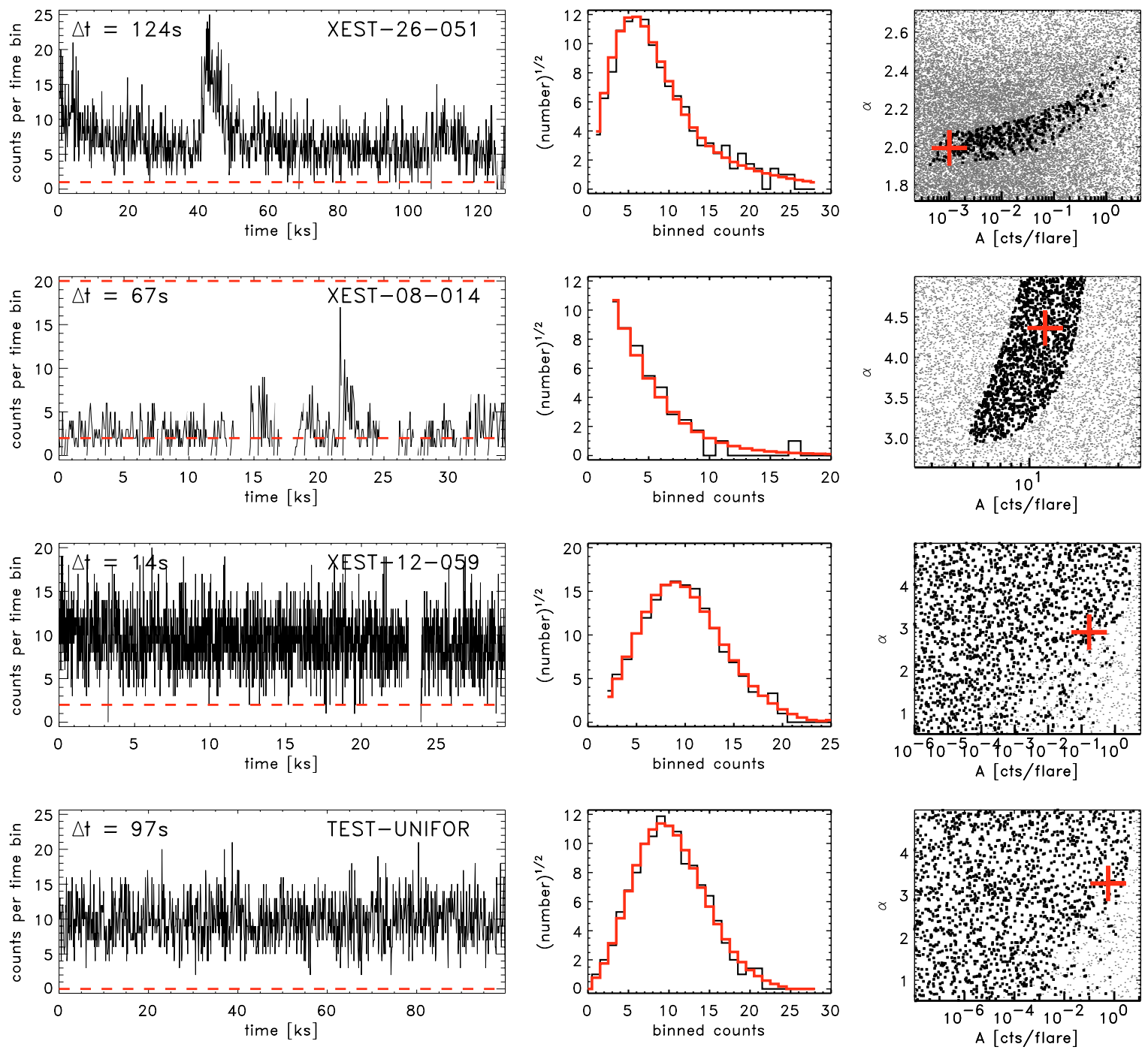

Fig. 4. Monte-Carlo exploration of three sample observations. One line of figures corresponds to one observation. Left column: light curves. Middle column: observed (black) and best-fit (red) histograms of the light curve values. Right column: $68 \%$ reduced acceptance regions in $(A, \alpha)$ space (projections from $(A, \alpha, B)$-space). $\alpha$ represents the power law slope of the flare amplitude distribution, and $A$ represents the lower cutoff of the flare amplitudes in cts/flare. Black dots represent points inside the $68 \%$ acceptance regions; gray dots represent point outside these regions; large red crosses denote the best-fit solutions. Note that due to the projection effects, black and gray dots may overlap. The last line represents a benchmark with uniform (quiescent) data.

cases the goodness-of-fit is questionable. A much safer result is possible for HD 285845, where $\alpha>3.5$ can firmly be established. In all other cases, $\alpha$ is unconstrained, or only constrained in combination with $A$ or $A / B$. The comparison with the pure Poisson noise example (Fig. 3 bottom) suggests that the observations from GK Tau, CoKu LkHa 332 G2, DN Tau, CoKu Tau/3, DI Tau, IT Tau, V773 Tau, XEST- 20-071, HL Tau, and V710 Tau are compatible with quiescent emission - a result which is also obvious from inspection of the light curves.

What can we conclude from the present study? Inspecting Table 1 and Figs. 4-A.3, we find the following:

- Most light curves studied here are compatible with a powerlaw distribution of the flare amplitudes (counts per flare in a fixed energy range). This confirms previous studies that have found such distributions for solar and stellar flares.

- In those cases where $\alpha$ could be constrained, its value exceeds 2. This is the case for 2MASS J04351316+2259, HD 29050, HQ Tau, HD 285845, and HD 31395.
- No clear distinction of accretors and non-accretors is possible.

- About half of the studied observations are compatible with a constant count rate at the given sensitivity, which may be due to their limited observing time of some $40 \mathrm{ks}$.

- From those targets where $\alpha$ and $A$ could be constrained, the following implications may be drawn. The observed values $\alpha \geq 2$ suggest the dominance of many small flares over few large flares in the coronal heating process, if we assume that the corona is heated by flares. Hence, our results support a stellar analog of the solar micro (or even nano-) flare heating scenario (Krucker \& Benz 1998), although at much higher flare energies than those of solar microflares. However, a clear statement can be made only for 4 main-sequence stars and one T Tauri star.

This present study complements a XEST investigation of individually detected flares by Stelzer et al. (2007), but uses a different approach because we are, in the present work, 
predominantly concerned with stochastic flaring and therefore with flare events that may not be detected individually in the light curves. Our conclusions are, however, compatible with the findings by Stelzer et al. (2007): They reported $\alpha=2.4 \pm 0.5$ for a TMC sample of T Tauri stars that showed detectable flares. The larger samples that can be accessed by our method is compromised by the weaker constraints in the statistical results. We also re-emphasize that a significant conclusion on $\alpha$ has been obtained for only one T Tauri star.

This result is analogous to findings from nearby active stars where a dominance of $\alpha$ values in the range of 2-3 has been found (Audard et al. 2000; Kashyap et al. 2002; Güdel et al. 2003; Arzner \& Güdel 2004). Although this points at important contributions of stochastic flares to coronal heating, this hypothesis cannot be fully proven using this methodology because our analysis requires the power-law distribution of the flare occurrence rate to continue to flares that cannot be individually detected in the light curves. The analogy of our findings with previously reported results for magnetically active stars suggests that X-ray sources in T Tauri stars, at least as far as the CCD detectors used here can record their X-ray emission, are compatible with a coronal model in which small flares play an important role.

We conclude with a few methodological remarks. In this work, we have assumed that the flare shape is constant. The dependence of the decay time on the flare size has been discussed in Güdel et al. (2003). Observations suggest that the decay time $\tau$ varies with the total number $N$ of counts according to $\tau \propto N^{\beta}$, where $\beta$ is no more than 0.25 (our current assumption of constant flare shapes amounts to setting $\beta=0$, an assumption also supported by Güdel et al. 2003). These authors found that $\alpha$ increases with increasing $\beta$. They interpreted this as being due to a larger time occupation by relatively large count rates. The light curve appears "softer", requiring a higher $\alpha$. As a consequence, also for our work, $\alpha$ can only increase if we allow larger flares to decay less rapidly.

For parametric flare amplitude distributions with few parameters, the parameters could also be estimated by forming intermediate statistics such as $\lambda\langle a\rangle$ (average count rate) and $\lambda\left\langle a^{2}\right\rangle$ (variance of the light curve), and then functionally relate these statistics to the model parameters. Such an approach is less optimal since it does not use the full shape of single-time distributions such as $P_{\delta}(n)$ and $P_{\delta}(x)$. Moreover, the intermediate statistics might not be sufficient and the computation of parameter errors is not as straightforward as from the Poisson likelihood. Since the evaluation of Eq. (6) is computationally not demanding, we argue that it should be used rather than some intermediate statistics in order to fully exploit the observed single-time statistics.

Acknowledgements. The authors thank the International Space Science Institute (ISSI) in Bern, Switzerland, for logistic and financial support during several XEST workshops. This research is based on observations obtained with $X M M-N e w t o n$, an ESA science mission with instruments and contributions directly funded by ESA member states and the USA (NASA). M.G. acknowledges support from the Swiss National Science Foundation under grant No. 20-66875. M.A. acknowledges support by National Aeronautics and Space Administration (NASA) grant NNG05GF92G. In addition, he acknowledges support from a Swiss National Science Foundation Professorship (PP002-110504).

\section{References}

Arzner, K., \& Güdel, M. 2004, ApJ, 602, 363

Audard, M., Güdel, M., \& Guinan, E. F. 1999, ApJ, 513, L53

Audard, M., Güdel, M., Drake, J. J., \& Kashyap, V. 2000, ApJ, 541, 396

Bondesson, L. 1988, Shot noise processes and distributions, in Encyclopedia of Statistical Science, Vol. 8 (New York: John Wiley)

Burge, R. E., Fiffy, M. A., Greenaway, A. H., \& Ross, G. 1974, J. Phys. D: Appl. Phys., 7, L61

Crosby, N. B., Aschwanden, M. J., \& Dennis, B. R. 1993, Sol. Phys., 143, 275

Feller, W. 1968, An introduction to probability theory and its applications (New York: John Wiley \& Sons)

Güdel, M. 2002, ARA\&A, 40, 217

Güdel, M., Audard, M., Kashyap, V. L., Drake, J. J., \& Guinan, E. F. 2003, ApJ, 582,423

Güdel, M. 2004, A\&AR, 12, 71

Güdel, M., Briggs, K. R., Arzner, K., et al. 2007, A\&A, 468, 353

Henery, R. J. 1984, J. Phys. A: Math Gen., 17, 3415

Hudson, H. S. 1991, Sol. Phys., 133, 357

Jansen, F., Lumb, D., Altieri, B., et al. 2001, A\&A, 365, L1

Kashyap, V., Drake, J. J., Güdel, M., \& Audard, M. 2002, ApJ, 580, 1118

Klibanov, M. V., Sacks, P. E., \& Tikhonravov, A. V. 1995, Inverse Problems, 11,1

Krucker, S., \& Benz, A. O. 1998, ApJ, 501, L213

Lee, J.-S. 1986, Opt. Eng., 25, 636

Lin, R. P., Schwartz, R. A., Kane, S. R., Pelling, R. M., \& Hurley, K. 1984, ApJ, 283, 421

Lukacs, E. 1970, Characteristic Functions (London: Griffin)

Mitra-Kraev, U., \& Benz, A. O. 2001, A\&A, 373, 318

Ness, J.-U., Güdel, M., Schmitt, J. H. M. M., Audard, M., \& Telleschi, A. 2004, A\&A, 427, 667

Parker, E. N. 1988, ApJ, 330, 474

Parnell, C. E., \& Jupp, P. E. 2000, ApJ, 529, 554

Reiss, R.-D. 1993, A course on Point processes, Springer Series in Statistics (Springer)

Scargle J. D. 1998, ApJ, 504, 405

Stelzer, B., Flaccomio, E., Briggs, K. R., et al. 2007, A\&A, 468, 463

Strüder, L., Briel, U., Dennerl, K., et al. 2001, A\&A, 365, L18

Turner, M. J. L., Abbey, A., Arnaud, M., et al. 2001, A\&A, 365, L27

Wolk, S. J., Harnden, F. R. Jr., Flaccomio, E., et al. 2005, ApJS, 160, 423 


\section{Online Material}




\section{Appendix A: A gallery of Monte-Carlo results}

Figures A.1 to A.3 provide an overview of all observations used in this study. These graphics represent the database for Table 1 .

The arrangement of the graphics as identical to Fig. 4. 
K. Arzner et al.: Superimposed flares, Online Material p 3
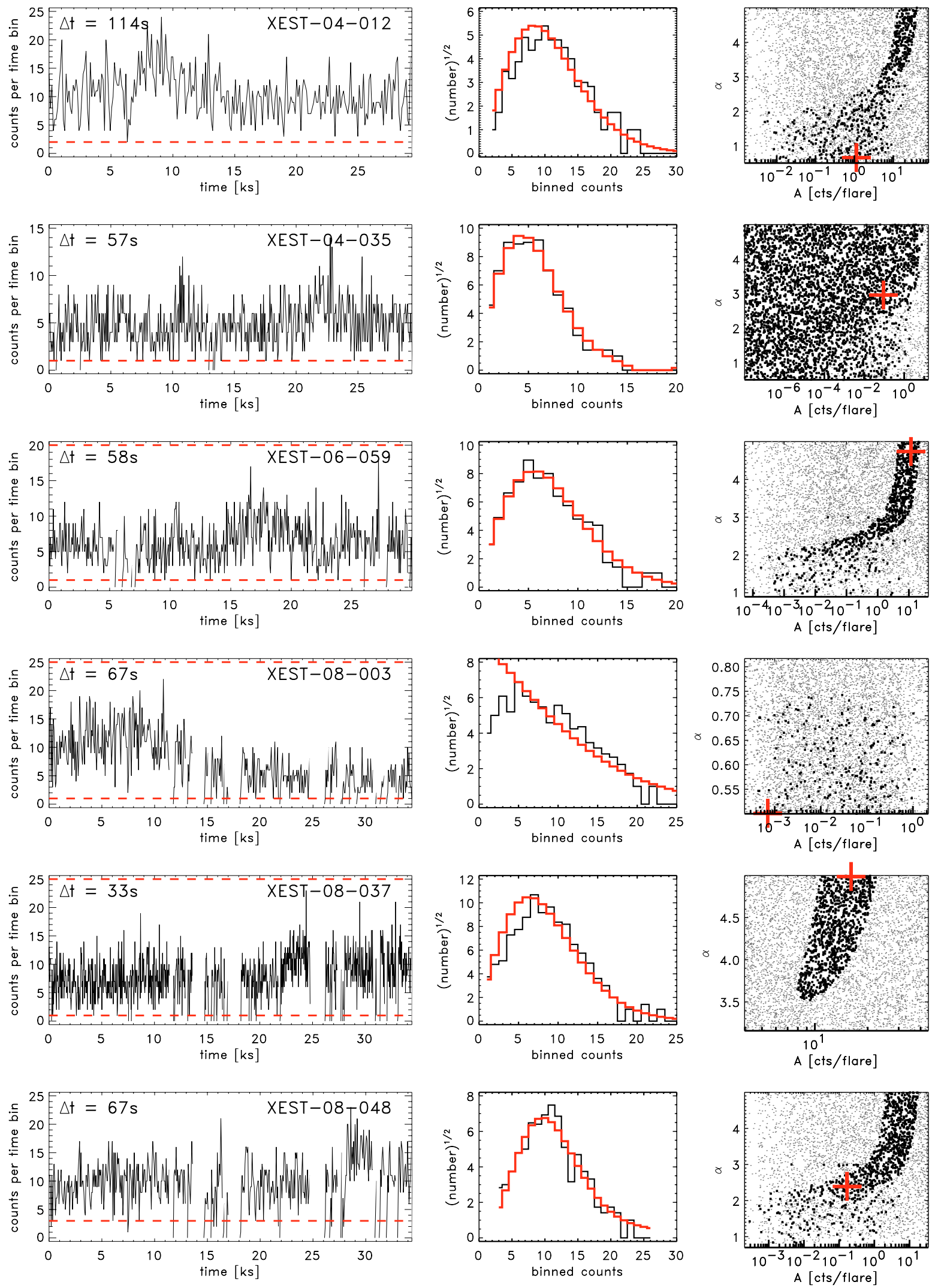

Fig. A.1. Continuation of Fig. 4. 
K. Arzner et al.: Superimposed flares, Online Material p 4
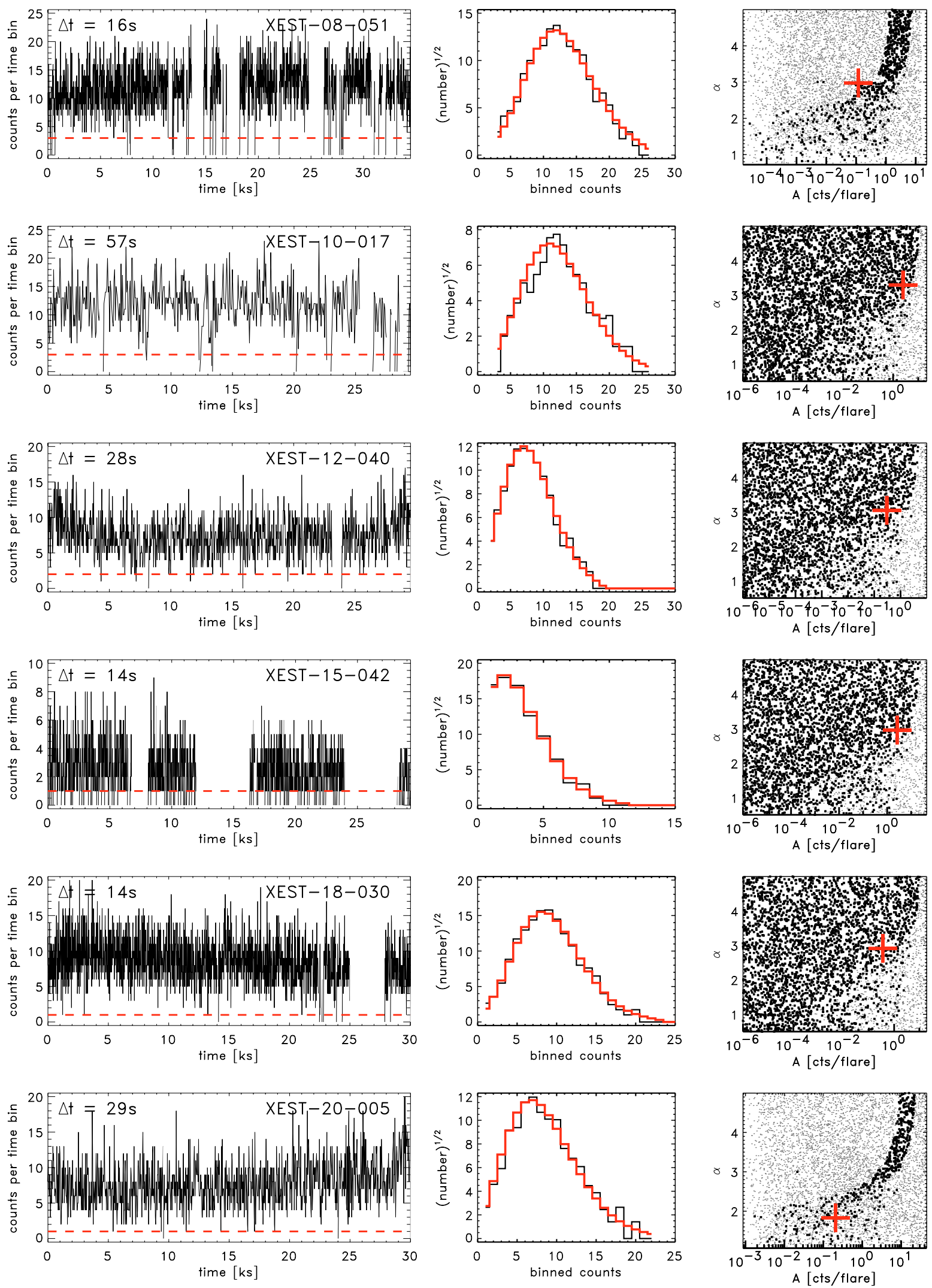

Fig. A.2. Continuation of Fig. A.1. 
K. Arzner et al.: Superimposed flares, Online Material p 5
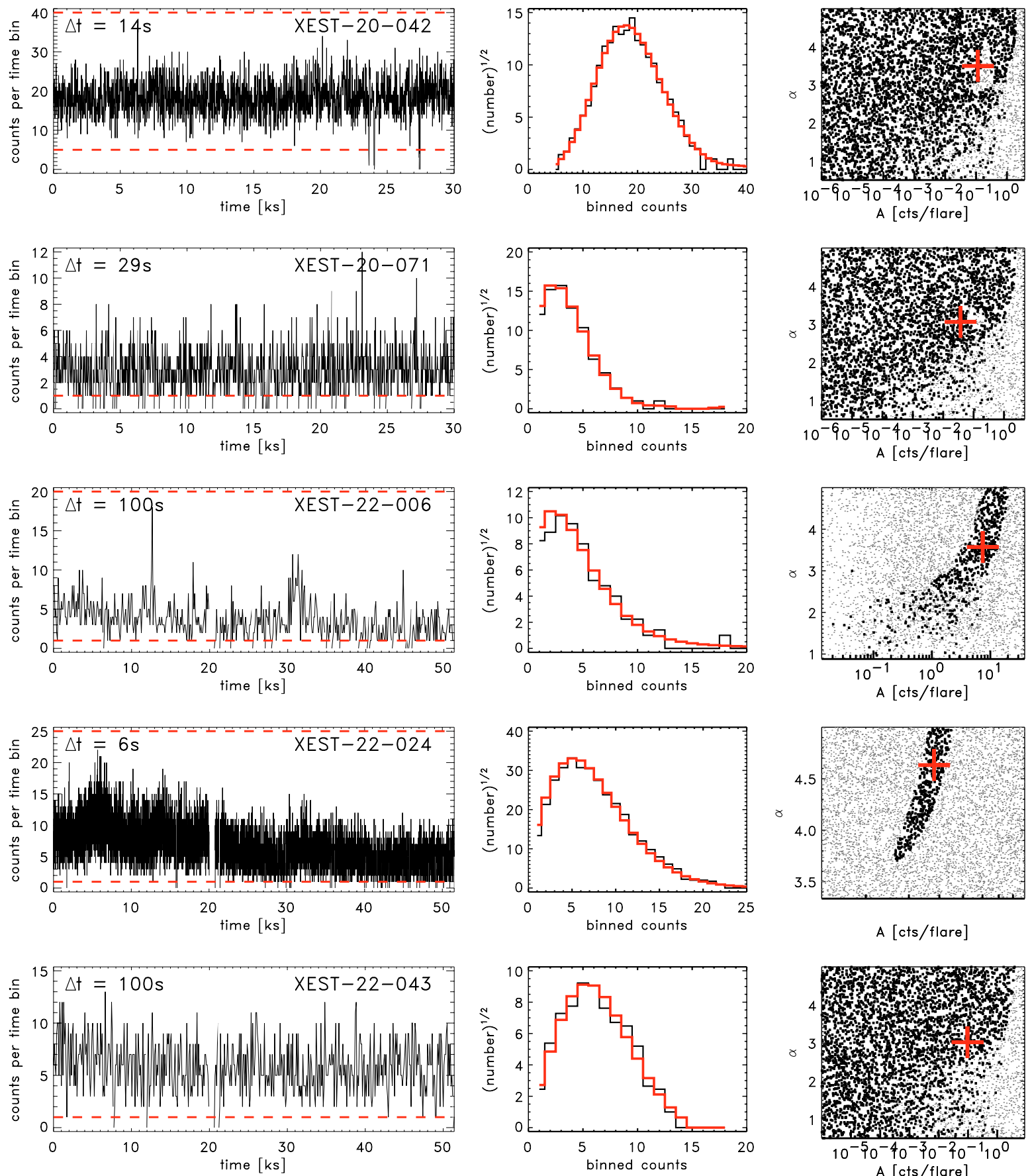

A [cts/flare]
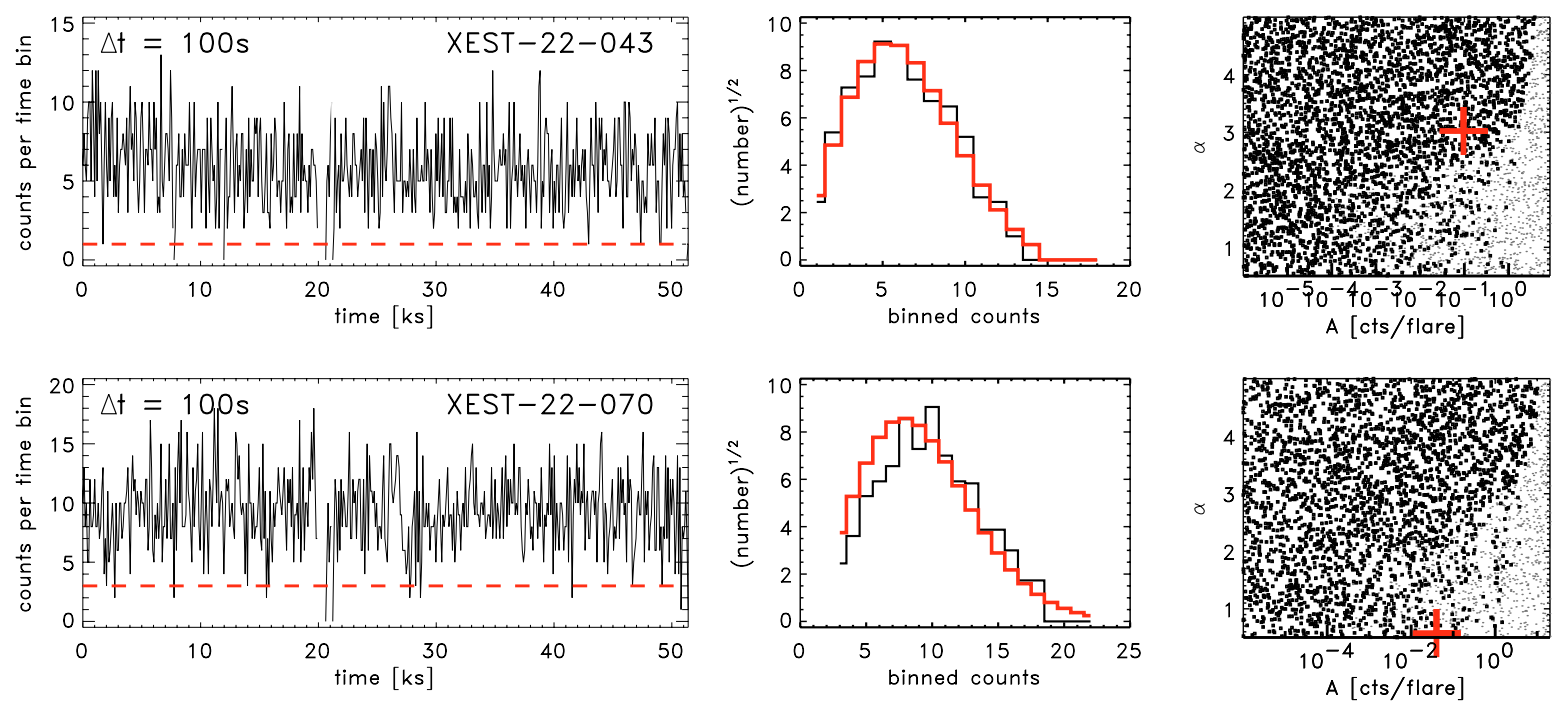

Fig. A.3. Continuation of Fig. A.2. 